American Journal of Pharmaceutical Education 2018; 82 (6) Article 6311.

\title{
RESEARCH
}

\section{Integration of the Pharmacists' Patient Care Process (PPCP) into a Comprehensive Disease Management Course Series}

\author{
Michael J. Gonyeau, PharmD, MEd, BSPharm, Margarita DiVall, PharmD, MEd, \\ Michael P. Conley, PharmD, Jason Lancaster, PharmD, MEd \\ Northeastern University, Bouvé College of Health Sciences, School of Pharmacy, Boston, Massachusetts \\ Submitted January 31, 2017; accepted June 13, 2017; published August 2018.
}

Objective. To implement and assess the curricular integration of the Pharmacists' Patient Care Process (PPCP) in a course series for second- and third-year pharmacy students.

Methods. The five-step PPCP was integrated within a four-semester pharmacotherapy course starting with the introductory course lectures. Beginning in the spring of 2015, the five steps of the PPCP were delivered to 129 P2 students, along with rollout of curricular integration within corresponding classroom and seminar activities and assessments. Integration focused on the development of course-specific lecture and seminar materials, a faculty guidance strategy and templates, and evaluation approaches for course assessments. Student comprehension and utilization of the PPCP were assessed via 61 unique assessments (12 examinations and 49 quizzes). Faculty incorporation and perception of the PPCP were evaluated via survey.

Results. Overall, students demonstrated the most understanding on the lowest levels of the PPCP: $83.6 \%$ and $82.2 \%$ for the Collect and Implement components, respectively, compared to the higherlevel components of Planning (78.0\%) and Follow-up (76.0\%). Faculty understanding, integration, and utilization of the PPCP in course materials were assessed approximately 6 months after implementation. Twenty-two faculty (96\% of course instructors) participated in the survey. Eighteen $(82 \%)$ have modified instructional materials to incorporate PPCP and among these, $89 \%$ agreed/ strongly agreed that they possessed a clear understanding of the PPCP.

Conclusion. Implementing a successful curricular change such as the integration of the PPCP across multiple courses requires a multi-faceted approach. The development of faculty templates and provision of support through various methods are necessary to ensure consistent and comprehensive integration across the curriculum. Additionally, evaluation of student performance and achievement of intended outcomes should be used to guide curricular assessments and continuous quality improvements throughout the process.

Keywords: JCPP, patient care process, curriculum, assessment

\section{INTRODUCTION}

The Joint Commission of Pharmacy Practitioners (JCPP) approved a newly developed description of the Pharmacists' Patient Care Process (PPCP) in May 2014, with the goal of improving patient health through integration of a comprehensive and consistent assessment process for pharmacists working as members of the health care team. ${ }^{1,2}$ The PPCP was developed by 11 national pharmacy organizations and describes the steps that pharmacists use to provide patient care services regardless of pharmacy practice setting or patient population. ${ }^{3}$ The

Corresponding Author: Michael J. Gonyeau, Northeastern University, Bouvé College of Health Sciences, School of Pharmacy, 360 Huntington Ave., R218, Boston, MA 02115. Tel: 617-373-5127.E-mail: m.gonyeau@northeastern.edu
PPCP focuses on five aspects of patient care based on the identified need for the profession to use as a consistent process for the delivery of patient care services. Its intent was to emphasize and contemporize pharmacists' role through a comprehensive approach to collaborative patientcentered care.

The American Association of Colleges of Pharmacy (AACP) Professional Affairs Standing Committee discussed the PPCP in its 2015 report, stating a need for assessment and advocacy of the process in practice, and the need for the academy to educate all student pharmacists to develop knowledge and skills related to the PPCP. ${ }^{4}$ The committee proposed the following policy statement related to the PPCP: "Policy Statement 3: Administrators, faculty members, preceptors and student pharmacists at all colleges and schools of pharmacy share 


\section{American Journal of Pharmaceutical Education 2018; 82 (6) Article 6311.}

responsibility for stimulating change in pharmacy practice consistent with the JCPP Vision for Pharmacy Practice and the Pharmacists' Patient Care Process."

The Accreditation Council for Pharmacy Education (ACPE) Standards 2016 have incorporated the PPCP into standard 10, stating that curricula need to prepare students to provide patient care as described in the PPCP. ${ }^{5}$ The standards state that the PPCP depends on the pharmacist establishing effective patient relationships, supporting appropriate and effective communication with the patients and their advocates. ${ }^{5}$ Thus all schools and colleges of pharmacy are required to integrate the PPCP into their curricula and demonstrate how students are learning this process and attaining competencies necessary to provide patient care. As of March 2017, only one publication has reported outcomes after implementation of the PPCP within a pharmacy curriculum. ${ }^{6}$ Rivkin described integration of the PPCP into a pharmacotherapy course taught to first-year pharmacy (P1) students. In this course, the PPCP was presented using a variety of techniques, including incorporation within patient cases and written work with learning outcomes assessed via examinations and a student survey.

The objectives of this study were to implement and assess the curricular integration of the Pharmacists' Patient Care Process (PPCP) in a course series for secondand third-year pharmacy (P2 and P3) students. This research article also describes Northeastern University School of Pharmacy's approach to faculty development in ensuring the consistent emphasis of PPCP throughout several courses.

\section{METHODS}

This project was approved by Northeastern University Institutional Review Board. In fall 2014, a longitudinal roll out and integration of the PPCP beginning with the Comprehensive Disease Management (CDM) course series was undertaken. The CDM series spans four consecutive semesters of the P2 and P3 years and integrates patient assessment, pathophysiology, selfcare, and therapeutics. Each semester, students take a large classroom (100-150 students) didactic course (6 semester hours) and small group ( $<19$ students) seminar (1semester hour) during which they work through cases and simulations, apply knowledge, and develop necessary skills and attitudes to prepare them for APPEs. In the last three semesters, students participate in the CDM Skills Lab where they practice and demonstrate skills required in pharmacy practice. The course series is team taught by faculty members in the Department of Pharmacy and Health Systems Sciences (DPHSS) who have expertise in a wide range of pharmacotherapy areas.
Eleven coordinators deliver content and activities across all courses and semesters.

The PPCP was introduced to P2 students as part of the introductory patient assessment lectures in the first CDM module. Integration of the key concepts surrounding patient assessment, documentation and identification of drug-related problems, and interpretation of clinical laboratory values lectures were reorganized to align with the PPCP. Traditionally, these materials were presented using various methods of patient assessment, namely the QuEST SCHOLAR-MAC (Quickly and accurately assess your patient; Establish whether patient is appropriate for self-care treatment; Suggest appropriate self-care strategies; Talk with your patient about those strategies. Symptoms, Characteristics, History, Onset, Location, Aggravating Factors, Remitting Factors, Medications, Allergies, Conditions) and SOAP (Subjective, Objective, Assessment, Plan). ${ }^{7,8}$ To facilitate this transition, a series of slides and graphics were developed to guide students through the various patient assessment approaches and explain exactly where and how they fit into the PPCP. A document explaining the Pharmacists' Patient Care Process was posted on the class Blackboard (Blackboard Inc, Washington, DC) site as a required reading.

A multi-faceted approach was used to ensure adequate emphasis of PPCP throughout the series. First, the existing CDM faculty guide that summarizes best practices for instructional design, objective development, and assessment was amended to incorporate the summary of the PPCP process and recommendations for integrating PPCP throughout didactic and active learning activities. Second, the importance of emphasizing PPCP was discussed at team faculty planning meetings that occur prior to the start of each semester. After the first semester, early adopters provided examples of how they integrated and emphasized PPCP in their instruction and active learning. For example, multiple faculty incorporated the PPCP into think-pair-share activities, mini case studies, audience response system questions, and game formats. A lecture PowerPoint (Microsoft, Seattle, WA) template was created to aid faculty in incorporating the PPCP into their didactic approach and class materials. A PPCP concept map was created and introduced to replace the old patient assessment worksheets (Appendix 1). The concept map was designed to guide students through a systematic thought process when evaluating patient care issues as part of a weekly seminar coursework. The concept map acts as a roadmap to aid student understanding and application of the PPCP and encourages development of therapeutic and critical thinking skills through work-up of patient cases. The concept map is color coded to correspond to the steps of PPCP and includes question prompts. 


\section{American Journal of Pharmaceutical Education 2018; 82 (6) Article 6311.}

Students submit completed concept maps as part of their seminar homework and receive individual feedback from facilitators to further enhance their skill development. Lastly, a series of department-wide faculty development sessions were held to discuss PPCP implementation.

PPCP was introduced to the entire department (with 23 instructors teaching within the CDM course series and 13 faculty teaching in other courses) at a departmental meeting at the end of the first semester of implementation. Faculty members in charge of introducing PPCP and early adopters provided an overview of PPCP and shared what students were learning and strategies for emphasizing PPCP through the remainder of the series. The goal was to encourage CDM series faculty to convert/integrate their current lecture and seminar materials that used the SOAP and QuEST SCHOLAR-MAC format to the PPCP. Opportunities for integration of PPCP in the P1 year were also discussed at this meeting.

At the beginning of the third semester of PPCP implementation, faculty members teaching in the series were reminded of the importance of integrating PPCP, available templates, and frequently used strategies. At the end of the third semester, faculty members were surveyed to determine their overall understanding of the PPCP, the depth and nature of adoption of the PPCP within the curriculum, and identify possible needs for future implementation and/or faculty development.

To evaluate student PPCP competency, attainment questions on each major assessment in the CDM series were categorized according to the five PPCP steps by the faculty authors. ExamSoft (ExamSoft, Dallas, TX) was used for all assessments in the series as it allows for questions and rubric dimensions to be mapped to multiple categories, including programmatic learning outcomes, topics, and other categories of interest. To ensure validity of mapping across all examinations in the four CDM modules, two faculty authors reviewed each question independently and a third reviewer served as arbitrator in cases of disagreement. Authors attempted to map each assessment question to one PPCP step based on the corresponding competencies. Examples of types of questions for each step are available in Table 1. Questions were dual coded (ie, more than one step assigned) if they specifically asked the student to provide two responses in their answer related to competencies falling within the different steps of the PPCP. In situations where students were asked to provide a therapeutic recommendation and a rationale for their selection, the questions were coded as both Assessment and Plan. The authors came to a consensus regarding "best practice" examples of questions that assessed each of the five PPCP steps and amended the school's ExamSoft categories guide to include PPCP category mapping recommendations and examples (Table 1). A faculty development session was held after the first cycle of the revised course series to discuss the results of the survey and ExamSoft assessment data, reflections on the first year of implementation, and the recommended mapping for the subsequent CDM course series.

\section{RESULTS}

Twenty-two faculty ( $96 \%$ of course instructors) responded to the web-based survey after three semesters. Results from this survey indicated that 19 faculty (86.4\%) attended at least one department meeting where the curricular PPCP integration was discussed and 14 faculty $(64 \%)$ attended both meetings. The majority of course instructors $(\mathrm{N}=18,82 \%)$ reported incorporating the PPCP into their instruction materials. Those who did not incorporate the PPCP into their materials reported that

Table 1. Examples of Assessment Question Mapping

\begin{tabular}{ll}
\hline Step & Types of Questions \\
\hline Collect & $\begin{array}{l}\text { Identify pertinent patient information necessary to resolve drug-related problems and basic calculation } \\
\text { (ie, creatinine clearance or ideal body weight). }\end{array}$ \\
Assess & $\begin{array}{l}\text { Evaluation of laboratory results, side effect profiles of different medications, rationales for therapy, } \\
\text { pathophysiology and drug mechanism of action. }\end{array}$
\end{tabular}

Plan Therapeutic recommendations (specific drug, dosing) that require the pharmacist to collaborate with another health care professional $+/$ - rationale for their selection.

Implement Patient education, communication between the pharmacist and another health care provider, documentation of a patient encounter, recommendation involving self-care/OTC or vaccination that could be initiated by a pharmacist without authorization or partnership with another health care provider.

Follow-up Monitoring parameters such as adverse drug events, patient follow up, and goals of therapy. 


\section{American Journal of Pharmaceutical Education 2018; 82 (6) Article 6311.}

they were not familiar enough with the PPCP to implement it at that time.

Table 3 presents the various methods by which faculty incorporated/reinforced the PPCP into their course materials. Most commonly, faculty referred to the PPCP during class (56\%), and developed active learning activities for classroom (44\%) and seminar (56\%) using PPCP. Out of 18 faculty, 5 (28\%) strongly agreed, 11 (61\%) agreed, and $2(11 \%)$ disagreed that they have a clear understanding of the specific abilities covered by each of the five steps of the PPCP (eg, I understand what skills and abilities student must possess to develop a Plan vs Implement). Out of the 22 faculty who completed the survey, $77 \%$ agreed that they would like to continue to participate in faculty development on integration of PPCP into the curriculum. Most frequently, faculty stated that additional examples of instructional materials (both didactic and experiential) and further review of specific competencies covered by the five PPCP steps will be helpful.

Over the first revised CDM series cycle, 110 students were evaluated using 61 summative assessments: 12 exams (approximately 70 points, 50 multiple choice and short answer questions, 2 hours in duration) and 49 quizzes (approximately 15 multiple choice questions, $20 \mathrm{~min}$ utes in duration). Individual assessment, overall course performance, and grade distribution for this cohort of students were similar to historical comparisons. Table 2 summarizes the distribution of the assessment questions categorized by PPCP step, and the descriptive characteristics of student achievement. Questions focusing on the Assess and Plan steps of the PPCP were most represented across all assessments, while the Collect and Follow-up steps were least represented. Student aggregate performance was highest for the Collect- and Implement-related questions (average of $83.6 \%$ and $82.2 \%$, respectively), while it was the lowest for the Plan- and Follow-uprelated questions (average of $78.0 \%$ and $76.0 \%$, respectively). Most notably, up to $12 \%$ and $16 \%$ of students, respectively, did not meet an acceptable threshold of competence (defined by an aggregate average of at least $70 \%$ ) for assessment questions categorized within the Plan and Follow-up steps.
Weekly homework submissions $(\mathrm{N}=32$ over four semesters) that included assignments such as progress notes, interventions forms, and completed PCPP concept maps, were assessed throughout the course series. These assignments were graded using electronic rubrics (Turnitin, Oakland, CA) integrated into the learning management system (Blackboard, Inc, Washington, DC). The aggregate average scores for the homework assessments ranged from 93.5 (5.3\%) during CDM 1 to 95.8 (3.7\%) in CDM 4. Individual assessment, overall course performance, and grade distribution for this cohort of students were similar to historical student cohorts in the CDM courses.

\section{DISCUSSION}

The overall goal of adopting the PPCP within this course series was to streamline and emphasize the approach to assessment, care and follow-up of patients across all populations in all health care settings. Enhancing student retention of these skills to a higher degree than had been done using previous methods of instruction was another goal. Prior to modification of the course curriculum, student feedback via end-of-semester evaluations indicated confusion regarding when SOAP process should be used vs QuEST-SCHOLAR MAC. The final aim was to ensure that both students and faculty were familiar with the modernized patient care process.

As part of the initial instruction and ongoing reinforcement of the PCPP, faculty members drew direct parallels to the other patient assessment methods (eg, SOAP, QuEST-SCHOLAR MAC) and emphasized how the PPCP serves as an overarching method to enhance and support the appropriate management of patient health and medication use. In addition to integrating this process within the beginning section of the disease state management modules, faculty members used various methods such as reinforcement in the classroom and activities designed for active learning in the classroom and in seminar. When examining competency achievement data, it can be noted that almost $70 \%$ of assessment questions were focused on the Assess and Plan phases of the PPCP. However, the data also indicate that there were more students who had aggregate scores below a $70 \%$ threshold for

Table 2. Competency Achievement Data ( $N=110$ students)

\begin{tabular}{|c|c|c|c|c|c|}
\hline PPCP Steps & Collect & Assess & Plan & Implement & Follow-up \\
\hline Items (n) & 94 & 487 & 286 & 151 & 91 \\
\hline Assessments (n) & 35 & 55 & 39 & 37 & 33 \\
\hline Range of aggregate scores per student (\%) & $62.2-94.7$ & $67.6-93.9$ & $63.1-91.1$ & $66.5-92.9$ & $61.2-96.1$ \\
\hline Students with aggregate score $<70 \%, \mathrm{n}(\%)$ & $1(0.9)$ & $1(0.9)$ & $13(11.8)$ & $6(5.5)$ & $18(16.4)$ \\
\hline
\end{tabular}

Abbreviations: $\mathrm{PPCP}=$ pharmacist patient care process 


\section{American Journal of Pharmaceutical Education 2018; 82 (6) Article 6311.}

Table 3. Responses to Faculty Survey Regarding PPCP Curriculum Integration $(\mathrm{N}=18)$, (faculty were allowed to check all that apply)

\begin{tabular}{lr}
\hline Answer & $\begin{array}{r}\text { Response } \\
\text { n (\%) }\end{array}$ \\
\hline Methods of incorporation of PPCP into instruction & $8(44)$ \\
Added an overview of PPCP to my lecture materials & $10(56)$ \\
Referred to the 5 components/steps of PPCP as I present content related to a disease state or teach & $8(44)$ \\
$\quad$ a specific skill set & $10(56)$ \\
Included active learning exercises that apply content covered using PPCP as part of the LECTURE & \\
$\quad$ component of the course & $6(33)$ \\
Included active learning exercises that apply content covered using PPCP as part of the SEMINAR & \\
$\quad$ component of the course & $6(33)$ \\
Reinforced PPCP during experiential learning (ie, APPE) & $12(67)$ \\
& $7(39)$ \\
Usefulness of faculty development materials available & $10(56)$ \\
$\quad$ CDM Faculty Guide & $10(56)$ \\
PPCP document & \\
$\quad$ Extroductory PPCP materials delivered at the start of the CDM series & \\
Discussions of how to integrate PPCP into instruction with one or more colleagues & \\
\hline
\end{tabular}

the competencies within the Plan step (12\%) and the Follow-up step $(17.5 \%)$. It is not surprising that, of the five steps of the PPCP, student performance was the highest on competencies covered by the Collect step, as these questions do not require any interpretation or decision-making. The assessment data were shared and discussed with course faculty. Faculty members agreed that while the Collect and Implement steps were also comprehensively assessed through seminar rubrics and homework submissions (eg, clinical intervention forms, progress notes, etc), the assessment of the Follow-up step should be more deliberate on quizzes and examinations. Finally, as the integration of PPCP into the curriculum expands into earlier courses, the opportunity for gathering assessment data on competencies related to the process will also expand.

While there are similarities to this study's approach with those of Rivkin's, there are several differences worth noting. ${ }^{6}$ At both schools, PPCP was integrated as part of a pharmacotherapy course or course series. Both schools used electronic polling, clinical case scenarios, and development of written work to facilitate student engagement. However, the faculty at Northeastern University School of Pharmacy went beyond the initial introduction of the PPCP and deliberately incorporated and emphasized the PPCP throughout the subsequent three semesters. To accomplish this, faculty development sessions were conducted and templates for faculty use were created. Finally, while Rivkin polled students to obtain their perceptions on personal development of confidence or competence in using the principles within the PPCP, the authors of this study were able to evaluate attainment of competencies covered by the five PPCP steps in a large number of assessments across four semesters. ${ }^{6}$ The assessment data were shared with the faculty and areas for improvement of integration and assessment of PPCP were identified.

This study has several limitations. First, both the categorization scheme for assessment questions and the faculty survey were developed by the faculty authors. While this approach provided an internal validation process, these items were not externally validated. Second, the survey approach provided a perspective from the majority of the faculty in the course series; however, a focus-group approach may have added more depth and understanding as to why not all of the faculty members were interested or able to include the PPCP within their instruction, and identify additional resources that may be helpful to them. Lastly, given the nature of the course series, which relies on multiple faculty to deliver the course content, inter-faculty differences can, and do, exist. Thus, despite oversight from a course coordinator, faculty may have interpreted the PPCP differently within their respective course materials.

Plans are underway to incorporate PPCP into the P1 courses (eg, communications can focus on Collect and Pharmacology can focus and emphasize Follow-Up). The importance of emphasizing PPCP throughout IPPE and APPE courses has also been recognized. To increase pharmacist awareness of the PPCP, appropriate preceptor development needs to be delivered.

Other pharmacy schools can adopt this multi-faceted approach of PPCP implementation without the need for 


\section{American Journal of Pharmaceutical Education 2018; 82 (6) Article 6311.}

extensive curricular review and/or overhaul. It would be of greatest benefit to work with faculty collectively, not only to ensure familiarity and uniformity with the various aspects and implementation of the PPCP, but also to identify an area where patient care skills are introduced, even if the students have not delved deeply into the pharmacologic/ non-pharmacologic management of various disease states. Early introduction of the PPCP would allow for increased levels of transferability of the skills required to perform the five steps in this process into numerous practice areas during IPPEs, regardless of whether students have been fully exposed to the therapeutic management of disease states, and this could be an area for future research. Furthermore, the impact of these skills can be assessed in numerous ways, including examinations, performancebased assessments, and using observation and reflection in the experiential setting. Faculty and preceptor development are necessary to ensure appropriate introduction, reinforcement and assessment of PPCP throughout the curriculum. Ongoing support and application of quality assurance principles will help identify early success and opportunities for improvement.

\section{CONCLUSION}

Implementing a successful curricular change, such as the integration of the PPCP requires a multi-faceted approach. The development of faculty templates and provision of support through various methods are necessary to ensure consistent and comprehensive integration across the curriculum. Additionally, evaluation of student performance and achievement of intended outcomes should be used to guide curricular assessments and continuous quality improvements throughout this process.

\section{REFERENCES}

1. American Pharmacists Association. Pharmacists' patient care process. https://www.pharmacist.com/sites/default/files/files/ PatientCareProcess.pdf. Accessed May 31, 2017.

2. Hepler CD, Strand LM. Opportunities and responsibilities in pharmaceutical care. Am J Hosp Pharm. 1990;47(3):533-543.

3. American Pharmacists Association. JCPP approves pharmacists' patient care process. https://jcpp.net/patient-care-process/. Accessed May 31, 2017.

4. Taylor CT, Adams AJ, Albert EL, et al. Report of the 2014-2015 Professional Affairs Standing Committee: producing practice-ready pharmacy graduates in an era of value-based health care. Am J Pharm Educ. 2015;79(8):Article S12.

5. Accreditation Council for Pharmacy Education. Accreditation standards and key elements for the professional program leading to the doctor of pharmacy degree. Standards 2016. https://www.acpeaccredit.org/pdf/Standards2016FINAL.pdf. Accessed May 31, 2017. 6. Rivkin A. Thinking clinically from the beginning: early introduction of the pharmacists' patient care process. Am J Pharm Educ. 2016;80(10):Article 164.

7. Leibowitz K, Ginsburg D. Counseling self-treating patients quickly and effectively. Proceedings of the APhA Inaugural SelfCare Institute; May 17-19, 2002; Chantilly, VA.

8. Buring SM, Kirby J, Conrad WF. A structured approach for teaching students to counsel self-care patients. Am J Pharm Educ. 2007;71(1):Article 8. 


\section{American Journal of Pharmaceutical Education 2018; 82 (6) Article 6311.}

Appendix 1. Concept Map

Disease State/Medical Condition:

Collect: Step 1: Knowledge of Disease State/Medical Condition

Epidemiology

Pathophysiology

Signs and symptoms

Diagnostic features

Treatment options (pharmacologic and non-pharmacologic)

Collect: Step 2: Review of Evidence-based Guidelines or Landmark Clinical Trials

What evidence is relevant to your patient assessment?

Collect:

Step 3: Patient-Specific Factors

Subjective:

Objective:

Past Medical History:

Social History:

Family History:

Non-drug allergies:

\section{Collect:}

Step 4: Medication History/Med Rec

Current RX Medications:

OTC/Herbals/Vitamins:

Alternative Medications (CAM):

Drug Allergies:
Assess:

Step 5: Set Patient-Specific Goals

Does the patient need therapy to reach goals and why?

Acute or chronic or both?

What is the urgency of beginning therapy?

What will treatment solve/prevent?

Is non-drug therapy available and appropriate?

Assess: Step 6: Identify Problems

Refer to DRP slides for specific examples (indication, effectiveness, safety, adherence/patient education)

Assess: Step 7A: Medication Assessment

Evaluate need for therapy

Evaluate current therapy

Utilize info from MedRec process

Is patient responding to therapy, having side effects, adherent?

Is patient using anything $(\mathrm{Rx} / \mathrm{OTC})$ to treat condition?

Current meds best suited for patient?

Correct dose? (age, wt, renal/hepatic function)

Appropriate dosage form, route, frequency?

Appropriate duration of therapy?

Evaluate all other therapy options

What other medications could be used to treat the problem?

How do they compare to the current therapy?

How do they compare to one another?

Which is best suited for the patient and why?

Why are the other therapies less suited or not recommended?

When comparing ALL options - compare and contrast:

SEEC Safety, Efficacy, Ease of use, Cost

(coverage by insurance)

Pros/cons of each option

Plan: Step 8: Plan (Problem List with Plan for Each Problem)

Develop treatment plan for all problems

Drugs to be avoided
Assess: Step 7B: Overall Patient Assessment

What recommendations are you considering? Why are you considering this? Why are you not considering something else?

Cite evidence-based arguments Incorporate patient-specific goals

How is your assessment impacted by other factors?

Other disease states

Other current medications

Patient and/or provider preferences

Insurance coverage 


\section{American Journal of Pharmaceutical Education 2018; 82 (6) Article 6311.}

Appendix 1. (Continued)

Step 9: Implement

Patient and provider education/communication/documentation

Anticipate any transitions of care issues

Consider and recommend any health preventive issues (ie, vaccines)

Consider with whom patient should follow up (eg, MD, RPh, others) and frequency of follow up

Follow-up: Step 10: Outcome Assessment and Monitoring Plan

Monitoring Parameters (toxic and therapeutic) 